\title{
Estado actual de la Psicología del Trabajo y las Organizaciones en México: un ensayo crítico.
}

\section{Actual situation of work/organizational psychology in Mexico: a critical essay.}

Fecha de recepción: 7-09-2014

Fecha de aceptación: 19-12-2014

\author{
Luis Fernando Arias Galicia \\ Centro de Investigación Transdisciplinar en Psicología \\ Universidad Autónoma del Estado de Morelos (México)
}

\section{resumen/abstract:}

Se proporciona una revisión crítica, desde el ángulo personal del autor, de la situación actual de la Psicología del Trabajo y las Organizaciones en México, a partir de 50 años de labor en esta área. Por tanto, se incluyen algunas pinceladas autobiográficas. Se comentan y critican las labores y las prácticas actuales de los psicólogos en este campo. Se incluyen ejemplos de las acciones profesionales deficientes y se coloca énfasis en las repercusiones éticas de las mismas y sus repercusiones negativas para las organizaciones y las personas. Con base en datos de noticias e investigaciones publicadas recientemente también se sugieren oportunidades para los profesionistas de estas áreas. Al final se ofrecen algunas sugerencias para los psicólogos a fin de incrementar la importancia de sus labores en las organizaciones.

A review is presented, from the author personal perspective, of the actual situation of work/organizational psychology in Mexico. This review is based on a 50 year experience working in this area. Therefore, some autobiographical anecdotes are included. Actual practices and tasks of psychologists in this field are presented and criticized. Examples of malpractices are included putting emphasis on the undesirable ethical and organizational consequences for persons and organizations. Opportunities for psychologists are discussed, taking as a basis some published research. Some suggestions targeted for psychologist are put forward in order to increase the value of their job for organizations.

\section{palabras clave/keywords:}

Psicología del trabajo y de las organizaciones; México; perspectiva actual

Work/organizational psychology; Mexico; actual perspective

\section{Introducción}

Con este ensayo intento ofrecer un panorama actual de la Psicología del Trabajo y las Organizaciones en México, aunque mi experiencia en varios países de Latinoamérica indica muchas similitudes. Debo decirlo: realmente dudé mucho. Tenía dos opciones: un artículo académico con profusión de citas (en caso de existir, lo cual dudo por las razones expuestas adelante) para fundamentar lo escrito o un ensayo en donde verter no sólo aspectos académicos sino también mis experiencias, inquietudes, angustias y conocimientos obtenidos a lo largo de cerca de 50 años de ejercicio en este campo en México (incluyendo la Administración del Capital Humano). Decidí tomar el segundo derrotero pues, pensé, me permitiría dar un toque más personal y, quizá, de mayor acercamiento a los posibles lectores. 
Así pues, no únicamente expreso aquí mis impresiones respecto a la situación actual del campo de la Psicología del Trabajo y las Organizaciones, y disciplinas conexas, sino salpico la narración con pinceladas autobiográficas a fin de dar mayor solidez a mis cogitaciones.

Inicio por recordar lo expresado por Kurt Lewin "No hay nada más práctico que una buena teoría", ampliado por Levi Leboyer: "No hay nada más teórico que una buena práctica"; debería establecerse, así, un maridaje indisoluble entre los aspectos conceptuales y el ejercicio cotidiano de la disciplina. Si he traído a colación las dos aseveraciones es para colocar énfasis en la pobreza de ambas situaciones en México.

Reconozco la proliferación de investigaciones en los últimos años referentes a las actitudes de los trabajadores, las cuales han seguido las modas en el ámbito internacional: satisfacción en el trabajo, compromiso, justicia, agotamiento ocupacional (burnout), acoso (mobbing), etc. Dichas pesquisas han sido llevadas a cabo primordialmente por académicos, las cuales han sido publicadas en diversas revistas nacionales e internacionales. (Un paréntesis: no existe una revista científica dedicada a la Psicología del Trabajo y las Organizaciones en México).

Una obra importante en este sentido es la coordinada por Arturo Juárez García (2014) Investigaciones psicométricas de factores psicosociales en trabajadores mexicanos, en la cual se incluye la fiabilidad y la validación de muy diversos instrumentos para analizar las actitudes en relación a temas tales como esfuerzo y recompensa, liderazgo, clima organizacional, violencia, etc.

La inmensa mayoría de las investigaciones mencionadas sólo constituyen diagnósticos en vez de intervenciones para intentar cambiar las situaciones encontradas. En cuanto a las empresas, la revista Expansión (2014c) publica los resultados de encuestas respecto a la cultura y el clima organizacionales, contribuyendo a la difusión del conocimiento. Empero, muchas empresas pagan buenas sumas a una organización lucrativa de cobertura internacional para aparecer en la lista de las mejores empresas para trabajar. Supuestamente se efectúan intervenciones para mejorar la calidad de vida de los trabajadores. No obstante, no se dan a conocer los avances fundamentados desde el ángulo metodológico, con lo cual pueden caer en la falacia "post hoc, ergo propter hoc" la cual consiste en confundir una relación temporal con una relación causal (Si se desea una exposición más amplia de este tema, consúltese mi libro de Metodología de la investigación, Arias Galicia, 2010).

No conozco publicaciones científicas donde se hayan efectuado intervenciones para mejorar dichas actitudes. Ciertamente, en la inmensa mayoría de las ocasiones la decisión de intervenir no está en las manos de los colegas sino de los directivos de las organizaciones, quienes, en términos generales, no consideran en sus presupuestos financieros dichas intervenciones. Por ende, la teoría no puede ser ampliada, pues falta la buena práctica al respecto.

\section{Divorcio entre psicólogos y directivos}

La situación se agrava. Los directivos y los psicólogos viven en mundos diferentes y, por ende, no se comunican, dado que no emplean el mismo lenguaje. Los primeros están interesados en cuestiones muy pragmáticas: producción, ventas, flujo de efectivo, cumplimiento 
de metas establecidas en los planes de la organización, costos, etc. Los segundos no han aprendido a mostrar cómo sus acciones pueden incidir sobre los temas de interés para los primeros. Parece ser éste un mal generalizado en el ámbito internacional, según percibo, si bien algunos países están más adelantados.

En mi experiencia personal, el hecho de haber cursado la Maestría en Administración en la Universidad de California, Berkeley, me abrió perspectivas insospechadas anteriormente. Emprendí estos estudios por mi interés en comprender mejor el funcionamiento de las organizaciones y su influencia sobre el comportamiento humano y viceversa. Gracias a una beca de la Universidad Nacional Autónoma de México y la Fundación Ford llevé a cabo estos cursos. El estudio de la contabilidad, las finanzas, la economía y disciplinas afines amplió considerablemente mis perspectivas y me permitió entender mejor la interacción entre los aspectos organizacionales y la conducta humana. Más adelante pondré ejemplos cómo he intentado romper el divorcio mencionado.

\section{La psicología ¿no necesita matemáticas?}

Antes de seguir adelante, manifiesto una hipótesis no comprobada metodológicamente sino sólo por observaciones informales: la mayoría de quienes deciden transitar por el camino de la Psicología toman esa decisión con base en una creencia: no se necesitan matemáticas. Esta ilusión se ve reforzada por las imágenes aparecidas en los filmes, la televisión, los diarios y otros medios en donde aparece el psicólogo indicando a una persona que se acueste y le cuente sus problemas. Así, pensar en estudiar estados financieros, informes de resultados, calcular los costos de la rotación de personal, el beneficio/costo de la capacitación y de las demás funciones del área de la psicología y del capital humano. etc. no cae dentro del campo de las expectativas de los psicólogos.

Aquí refiero una anécdota trágica. En un curso sobre Capacitación en el nivel de Master, en el cual incluyo un método para calcular el beneficio/costo de estas acciones a fin de poder mostrar a los directivos la potencial buena inversión representada por la capacitación, encontré que de los doce integrantes del grupo sólo una persona (proveniente del campo administrativo) pudo calcular acertadamente algo tan sencillo como un porcentaje. La importancia no estuvo en el simple cálculo, lo trascendente se refirió a la toma de decisiones costosas para la organización. Afortunadamente sólo se trató de un ejercicio; si algo semejante ocurriera en la vida real, los psicólogos se verían en serios problemas.

\section{Carencia de evaluaciones}

Esta es una situación angustiosa para mí. Veo mucha acción; pero sin evaluación de los resultados y por ende, no hay manera de mostrar a los directivos la valía del trabajo de los psicólogos. Sin embargo, esta deficiencia no es sólo de los colegas, sino de muchas personas, incluyendo a los directivos. Muchas decisiones se toman basándose en creencias en vez de conocimiento. En este tenor, cuando he podido efectuar evaluaciones siempre he encontrado que las decisiones se habían tomado en el sentido equivocado.

Para ilustrar lo anterior sólo mencionaré dos casos de mi experiencia. En una empresa comercial que contaba con unas 40 sucursales en la Ciudad de México, me pidieron asesoría,

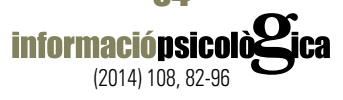


ya que tenían altos porcentajes de rotación externa. Decidí ir a los archivos para analizar algunas características de quienes habían renunciado (edad, distancia del centro de trabajo al domicilio, escolaridad, etc.). Aquí menciono un punto trascendente: los archivos contienen información valiosísima; constituyen verdaderos filones inexplorados de diamantes (ahora ya hay programas informáticos para efectuar la denominada "minería de datos"; pero serán inútiles si no hay espíritu investigador y evaluación de resultados). En esa empresa el director general estableció una política de contratar a personas jóvenes de sexo femenino. El razonamiento fue: "si un cliente es atendido por una muchacha de buen ver, volverá a comprar". Por la rotación existente, quedaban vacantes, las cuales en ocasiones eran cubiertas por señoras de más de 40 años; pero eso no era del agrado del director. Después de analizar los datos, surgió un hecho: quienes duraban menos en la empresa y vendían poco eran las mujeres jóvenes; en cambio las señoras permanecían por más tiempo y vendían más. Por esa creencia infundada, los costos de operación resultaban estratosféricos, según pude demostrar. Así las decisiones eran inversas a lo conveniente.

Otro ejemplo lo constituye una empresa donde se fabricaba y vendían productos de cierta complejidad. Tenía más de 40 sucursales en todo el país. Debido a la naturaleza de los productos, era necesario capacitar a los vendedores durante un mes en el centro ad-hoc de la empresa situado en la capital. También sufría la empresa de un alto índice de rotación de este personal. El sistema de selección consistía en la aplicación de unas pruebas importadas de Alemania, además de entrevistas y otras técnicas. Las habían empleado los psicólogos de la empresa durante más de nueve años. Si se efectúan algunos cálculos sencillos se apreciará la magnitud financiera de las decisiones. Durante la capacitación era necesario pagar los salarios respectivos, además de los gastos de traslado de muy diversas ciudades del país, alojamiento, alimentación, lavado de ropa, etc. Además, dentro de los costos es necesario agregar el salario de los capacitadores, los gastos de materiales, electricidad, renta del local y así sucesivamente. Se contrataban alrededor de 200 vendedores anualmente o sea 1,800 vendedores aproximadamente en los nueve años. Si se multiplica esta cifra por los costos individuales de capacitación se tendrán cifras astronómicas. Sin embargo, nadie se había asomado a verificar la validez predictiva de los tests. Se prefería contratar a los mejor calificados en las mencionadas pruebas; ya que era "lógico" su mejor desempeño en el trabajo. Como había rotación y era necesario cubrir plazas, en ocasiones se contrataba a quienes obtenían puntuaciones bajas y medianas. Nuevamente recurrí a los archivos. Resultado: quienes denotaban puntuaciones bajas duraban poco tiempo en la empresa y vendían menos al igual que quienes salían mejor calificados (los preferidos). En otras palabras, por una creencia y una falta de verificación los costos eran enormes, además de propiciar la rotación y las menores ventas.

Esta carencia de comprobación de la idoneidad de las acciones me inquieta sobremanera. Las decisiones equivocadas no sólo tienen repercusiones en los aspectos financieros y operativos de las organizaciones sino, peor aún, en las vidas de las personas, ya que el fracaso en un empleo puede tener agudas consecuencias psicológicas y sociales. Se cometen, entonces, graves faltas éticas por parte de los colegas al no verificar la valía de sus acciones. 


\section{Problemáticas en la Selección de Personal}

Es muy común copiar instrumentos para emplearlos en la selección de personal. Se han empleado durante décadas tests generales (Raven, Dominoes, Army Beta, el MMPI, el Barsit, las técnicas proyectivas, etc). Dichos tests fueron diseñados para otros propósitos, primordialmente académicos, en vez de tener en mente las necesidades de las organizaciones. Además, fueron elaborados en culturas diferentes a la mexicana. Últimamente, inclusive se han colocado en sistemas informatizados; empero no es común dedicar tiempo y esfuerzo a su validación, fiabilidad y estandarización. También en algunas empresas se han utilizado la fisiognomonía (o sea deducir el comportamiento a partir de los rasgos faciales), la grafología, la astrología, etc.

De hecho, la Psicología del Trabajo y las Organizaciones parece tener una larga historia en México. En efecto, siguiendo mi afición a hurgar en la librerías "de viejo", encontré en una ocasión dos libritos fechados en la década de los años 20 del siglo anterior, en los que se daban detalles de la aplicación del test de Terman y Merril para la selección de policías y bomberos. Hay una laguna temporal en el desenvolvimiento de esta disciplina la cual no he podido completar. Nótese: se trataba de instrumentos creados fuera del país.

Como parte de mi inconformidad respecto al empleo de tests diseñados para áreas ajenas al trabajo, por una parte y, por la otra, proyectados en otras culturas, diseñé algunas pruebas adecuadas a los trabajos de oficina. Por ejemplo, comparación de números y comparación de nombres, ambas competencias resultan importantes en las labores oficinescas. Además, diseñé también una prueba de inteligencia con el mismo propósito. Dichos instrumentos los dejé en la empresa donde laboraba entonces e ignoro si los siguen utilizando.

\section{Lanzamiento de una empresa especializada}

En una ocasión asistí a un congreso internacional en el que se presentó una ponencia respecto a tests ingleses elaborados específicamente para empleo en las organizaciones, en diversos tipos de ocupaciones y adecuados a diversos niveles organizacionales. Me interesaron sobremanera y más cuando los ponentes me indicaron la existencia de muchos de esos instrumentos en español y, además, colocados en sistemas de cómputo. Como continuación, se decidió crear en México una filial de esa empresa. Con un equipo formado por tres personas iniciamos la promoción. Inmediatamente nos enfrentamos al rechazo, ya que, como he mencionado antes, en muchas empresas se acostumbra la copia de tests, lo cual resulta gratis aparentemente porque no se contemplan los costos humanos y financieros de las decisiones equivocadas.

No obstante, empezamos a abrir mercado, especialmente en empresas inglesas asentadas en el país. Muchas de las nacionales preferían la práctica de la copia mientras un gran número de las norteamericanas se basaban fundamentalmente en la entrevista: en los Estados Unidos es necesario demostrar la valía de los tests para las contrataciones; por tanto, las empresas pueden enfrentar juicios legales en caso de no poder demostrar la validez.

\section{Fracaso de la empresa}

Después de algunos años, cuando ya empezábamos a tener pequeñas utilidades, la matriz londinense decidió la contratación de un nuevo director para el continente americano. Me

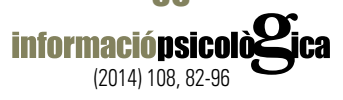


ofrecieron un magnífico sueldo mensual; pero adquiría el compromiso de vender un millón de dólares el primer año; dos millones, el segundo; y tres, a partir del tercero. Conociendo la situación en este país y la costumbre de copiar indiscriminadamente las pruebas, no acepté y, además, previne un posible fracaso por la mencionada costumbre de calcar los tests. Entonces el nuevo director contrató a otro gerente y a vendedores en Monterrey y Guadalajara además de la Ciudad de México y montó oficinas lujosas en las tres ciudades. Al cabo de un año perdió seis millones de dólares porque cada uno de los vendedores no aportó un solo peso. Como consecuencia, la filial mexicana desapareció. Este es otro ejemplo trágico de cómo la toma de decisiones basadas en creencias y no en conocimiento acarrea consecuencias funestas.

\section{Deficiencias en capital humano}

En esa empresa me llevé también sorpresas muy desagradables: existía una prueba con 60 ítems para tomar decisiones basadas en datos estadísticos, financieros, de producción, etc.; es decir, apoyadas en el análisis de datos numéricos. Se aplicaba a gerentes para selección o promoción. El promedio de aciertos en Inglaterra era algo así como de 50, en Francia era de 45 según mi memoria. En una reunión anual en Londres presenté los resultados de los gerentes mexicanos: 16 aciertos en promedio; no me creyeron y me pidieron revisar, lo cual hice y confirmé los resultados; hubo gerentes que no tuvieron un solo ítem respondido correctamente. Años después, con los resultados de las pruebas PISA comprobé los funestos desenlaces. Me apena y me duele la baja competitividad, en general, de nuestros directivos y de nuestros trabajadores.

\section{Un test fantasma}

La selección de personal es una de las áreas tradicionalmente ocupada por los psicólogos. Como he mencionado, se cometen graves errores y faltas éticas en este campo, puesto que muchos de los tests empleados no han sido validados ni cuentan con una adecuada fiabilidad y, como mucho, se calculan únicamente los baremos. Insisto nuevamente: es muy posible tomar malas decisiones.

Por ejemplo, un instrumento muy empleado durante años ha sido el Cleaver. En primer lugar, hasta donde percibo, existen varias versiones debido a diferentes traducciones, con lo que las versiones no concuerdan. Por otro lado, una búsqueda en la prestigiosa base internacional de artículos EBSCO no mostró ningún artículo científico en psicología que incluyera el término Cleaver en el título. Lo mismo sucedió en una búsqueda en la base Scielo, la cual incluye preponderantemente artículos en español y portugués. Por tanto, es un instrumento que aparentemente sólo se usa en México. Para agravar la situación en una investigación llevada a cabo entre 180 profesores de una institución educativa privada de nivel superior, Rojas Sierra y Acevedo Rocha (1998) encontraron diferencias marcadas entre las normas supuestamente universales de esta herramienta y los resultados obtenidos en las muestras mexicanas, apuntando nuevamente a una inadecuada toma de decisiones y a faltas éticas de gravedad. 


\section{¿Todos los psicólogos pueden hacer selección de Personal?}

Quizá uno de los problemas estriba en la falta de conocimiento de los directivos respecto a los campos de la psicología; pues, basta con el hecho de que alguien tenga el título o esté estudiando para pensar en su idoneidad para seleccionar, capacitar al personal, etc. Así, me he topado con colegas cuya área de interés y de especialidad es la clínica y, sin embargo, son responsables de la selección, la cual llevan a cabo con criterios de esa rama en vez de la referente al trabajo. Contaré una anécdota para ilustrar la desviación de criterios. Al comienzo de mi trabajo en el área en una institución bancaria, la jefa de la sección, a quien llamaré C., era psicoanalista ortodoxa. En una ocasión llegó un joven muy limpio para solicitar un puesto como mozo, es decir, sin grandes demandas de tipo intelectual. Tendría aproximadamente unos 20 años y provenía de una familia de campesinos. Pasó las pruebas y la entrevista. Como parecía un buen candidato, se le envió al examen médico y a la encuesta económico-social, en la cual se va al domicilio del candidato a fin de verificar algunos puntos de su solicitud. Al poco tiempo, al pasar junto al escritorio de C., vi el expediente y la leyenda "Rechazado" con letras rojas. Me sorprendió y pregunté a C. las razones; ya que ella era quien tomaba la decisión final. Me espetó “¿No viste cuál es su pasatiempo favorito?". Sí, lo había visto: era "ver a las vacas", lo cual no me extrañó dada su composición familiar y su extracción campesina; supuse que se refería a atenderlas y cuidarlas. C. me preguntó con asombro “¿No te das cuenta de su fijación materna”? y agregó "Una persona así no debe pertenecer a esta empresa", lo cual me pareció totalmente exagerado. A lo largo de mi carrera, he visto otras decisiones basadas en aspectos clínicos no relacionados directamente con el trabajo.

\section{La capacitación}

Ahora bien, el problema de la falta de talentos no es privativo de México. Según una investigación de Gran Thorton (2014), el 30\% de los directivos en el ámbito global indicaron la falta de personal cualificado como un freno en el crecimiento de sus empresas. Se presenta así, otra vez, la necesidad de capacitación y un filón para los psicólogos.

Respecto a la capacitación, existe una confusión gigantesca: equiparar dar cursos con capacitar o formar. Este concepto se refiere a fomentar las competencias en las personas, las cuales deberían estar relacionadas con el trabajo a desempeñar. Inclusive la Ley Federal del Trabajo en su artículo 153-A indica "Los patrones tienen la obligación de proporcionar a todos los trabajadores, y éstos a recibir, la capacitación o el adiestramiento en su trabajo que le permita elevar su nivel de vida, su competencia laboral y su productividad" (énfasis mío). Desgraciadamente, la misma Ley fomenta la confusión mencionada; ya que obliga a registrar los planes de capacitación para todos los trabajadores de la empresa (necesiten o no de formación) y a seguir toda una serie de trámites burocráticos al respecto. Entonces, un medio para elevar la calidad de vida y la productividad se convierte en un fin.

Aquí narro tres anécdotas. En una ocasión me llamaron de una empresa y me preguntaron qué cursos podría impartir. Inquirí sobre sus necesidades; la respuesta fue: "Ya estamos en septiembre y todavía tenemos mucho presupuesto sin utilizar; si no lo empleamos este año, el siguiente nos darán menos fondos". Sí, su necesidad era gastar dinero, en vez de capacitar.

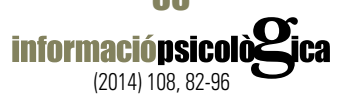


Otra anécdota: de otra empresa me llamaron para dar un curso; nuevamente, inquirí sobre sus necesidades. La respuesta fue "Vamos a tener una convención de gerentes y necesitamos un evento muy dinámico"; otra vez insistí en las necesidades detectadas de capacitación y la respuesta fue por el mismo tenor: "Necesitamos un curso con muchas técnicas grupales". Mi contestación fue "Entonces, no desean capacitar a los gerentes, sino divertirlos; mejor contraten a una bailarina, lo hará con más gracia que yo". La tercera anécdota. En un congreso asistí a una sesión donde se llevó a cabo una dinámica grupal, después de la misma el conductor indicó pasar a otra. Yo inquirí: “¿Cuál fue el aprendizaje dejado por la dinámica anterior? ¿Cuál fue la competencia adquirida?”. El conductor (que no capacitador) no respondió y siguió adelante con la nueva técnica grupal. Abandoné inmediatamente el salón.

Aquí existe otra confusión enorme: muchas veces los mejores "capacitadores" son quienes cuentan muchos chistes, son carismáticos y emplean muchas dinámicas grupales. De ninguna manera estoy en contra de estas acciones (yo mismo las utilizo); pero no estoy de acuerdo en centrar el evento en estos apoyos didácticos, olvidando la adquisición de competencias. Otra vez, se confunde el medio con el fin.

De acuerdo a la reciente Encuesta de Competencias profesionales (Centro de Investigación para el Desarrollo, A.C., 2014), el 70.54\% de los candidatos a un puesto no tenía los conocimientos necesarios; es decir, se presentó una brecha entre los requerimientos de los empleadores y la oferta. Esta encuesta se llevó a cabo en todos los estados de México, entre 481 empresas representativas de todos los sectores de actividad económica. Este dato implica la separación existente entre el sector educativo y el productivo, por una parte, y por la otra la necesidad de capacitación o formación, pues en múltiples ocasiones las empresas contratan a personas sin todas las competencias necesarias a fin de cubrir las vacantes existentes.

Otro caso: existe una brecha entre las necesidades de las empresas y la preparación académica de los ingenieros y los técnicos la cual debe llenarse mediante la capacitación, pese a la graduación de alrededor de 113,000 de estos profesionales al año. Afirmó Oscar Albín, Presidente Ejecutivo de la Industria de Autopartes: "Hoy en día cuando hay un ingeniero o técnico recién egresado, la empresa que lo contrata le tiene que dar un entrenamiento de entre seis y 18 meses antes de 'soltarle' una línea de prensas, una de inyección de plástico o un robot" (2013).

En los últimos tiempos se han establecido en México plantas armadoras de autos, además de las ya existentes. La finalidad consiste en surtir al mercado norteamericano primordialmente, aprovechando el hecho de compartir más de 3,000 kilómetros de frontera. Entonces, los psicólogos pueden trabajar al unísono con los ingenieros para apresurar la capacitación de los técnicos requeridos. Algo semejante está ocurriendo con la industria aeronáutica la cual ha centrado sus bases en el centro del país.

Aquí debo confesar una gran desilusión: precisamente por mi tendencia a dar solidez a las acciones y poder mostrar a los directivos la valía de la capacitación, diseñé un método para detectar necesidades ligadas a las competencias requeridas en el trabajo y evaluar los beneficios económicos de la capacitación. Escribí un libro al respecto, publicado en 1994, cuando fui Vice-Presidente de la Asociación Mexicana de Capacitación de Personal. Supuse una 
gran acogida a la obra, ya que proporciona herramientas a los capacitadores para mostrar a los directivos las repercusiones financieras favorables del proceso de capacitación si se lleva a cabo de una manera sistemática, desde la detección de necesidades ligadas al trabajo, hasta la determinación del beneficio/costo. Muy pocas personas se interesaron. Después, cuando fui Presidente de la misma asociación intenté capacitar a los capacitadores en este sistema; pero fracasé pues el número reducido de profesionales interesados indicó no ser un tema importante para ellos.

La cantidad de capacitadores con decisión de evaluar sus acciones puede contarse con los dedos de las dos manos (y sobran dedos). Estas experiencias me han llevado a la conclusión de la existencia de la gran confusión anotada antes: dar cursos y llevar a cabo dinámicas grupales es igual a capacitar, lo cual, naturalmente, es una fantasía. Igualmente, como en la determinación del beneficio/costo es necesario efectuar operaciones aritméticas sencillas lo cual se constituye en un tabú para los psicólogos y otros profesionistas de disciplinas relacionadas.

Según una investigación del Banco Interamericano de Desarrollo (2014) efectuada en nuestros países sólo el $12 \%$ de los empleadores no tuvo dificultades para encontrar trabajadores jóvenes con las habilidades requeridas por su empresa. Por una parte este dato señala la necesidad de un acercamiento entre las instituciones educativas y las fuentes de empleo; y por otro abre las puertas a los capacitadores para inducir las competencias necesarias mediante la formación dirigida al trabajo.

Mi deseo es muy intenso de un cambio paulatino en la situación actual por las nuevas disposiciones legales. En efecto, la Ley Federal del Trabajo establece en su artículo 153-K VI. "Evaluar los efectos de las acciones de capacitación y adiestramiento en la productividad dentro de las ramas industriales o actividades específicas de que se trate". Este punto deberá ser llevado a cabo por el Comité Nacional de Productividad. Así, se establece un mandamiento legal para la tasación de los resultados. Empero, tengo los pies sobre la tierra y me refreno un tanto en cuanto a mi entusiasmo por esta expectativa dadas dos razones:

- La muy débil inclinación de los psicólogos, profesionistas afines y directivos a verificar la efectividad de sus acciones.

- Muchas comisiones se forman teñidas de asuntos políticos en vez de técnicos o científicos.

El rápido cambio tecnológico demanda trabajadores con mayor educación formal, además de requerir capacitación frecuente. En una investigación efectuada por López-Acevedo, Tinajero y Rubio (2005) y auspiciada por el Banco Mundial se encontró, en México, una clara relación entre el nivel educativo y la capacitación de los trabajadores con el incremento en la productividad, aunque éste fue más notorio en el caso de la educación formal; en la misma pesquisa, los salarios mostraron aumentar conforme a la escolaridad. Esta investigación tomó como base la Encuesta Nacional de Empleo, Salarios, y Capacitación, llevada a cabo por el Instituto Nacional de Estadística, Geografía e Informática (INEGI) efectuada en 2001 entre 8,179 empresas comprendiendo varios sectores productivos así como diversos tamaños de organizaciones.

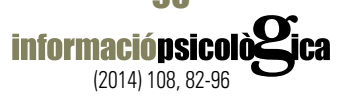


Es bien sabido que la Organización para la Cooperación y el Desarrollo Económico periódicamente mide, mediante las pruebas PISA, las habilidades en cuanto a la comprensión de lectura, la habilidad en el empleo de la aritmética y de principios científicos entre niños de primaria y secundaria. Hace lo mismo en relación a la población adulta. En una de las últimas investigaciones al respecto, se encontró una tendencia diáfana en cuanto a la relación entre dichas habilidades y la probabilidad de contar con un empleo, así como con el salario devengado, lo cual fue más notorio en cuanto al creciente empleo de las tecnologías de la información. Nuevamente, los capacitadores tienen una brillante oportunidad al respecto. En esta pesquisa participaron 24 países, con excepción de los Latinoamericanos (Quintini, 2014).

\section{La patología burocrática}

Volviendo al tema central, desde mi ángulo, los colegas en el área de Psicología del Trabajo y las Organizaciones y disciplinas afines han caído en la llamada "patología burocrática", la cual consiste en un desplazamiento de las metas; se pierde de vista el objetivo principal (en este caso, elevar la productividad y la calidad de vida) mediante las acciones cotidianas, los trámites y los procedimientos; entonces, los medios se convierten en un fin en sí mismos. De esta forma, la aplicación de pruebas, la realización de entrevistas, los cursos de capacitación, etc. devienen en el objetivo del trabajo de los colegas y se hace a un lado la productividad y el bienestar de los trabajadores.

Desgraciadamente, muchos psicólogos se han centrado en las funciones tradicionales: selección, capacitación y desarrollo (aunque tengo dudas respecto a la efectividad de sus acciones, por la patología mencionada en el párrafo previo), dejando en manos de otros profesionistas áreas importantes en la actualidad. Por ejemplo, desde décadas atrás se implantaron los sistemas de calidad total, lo cual significa un cambio en la cultura organizacional; empero, en vez de constituir un trabajo conjunto con los ingenieros, los psicólogos han abdicado de favor de aquéllos.

En la actualidad se menciona, y ya se trata de una moda, el tema de la responsabilidad social, la cual implica, entre otros aspectos, no sólo el cumplimiento de la legislación laboral sino la percepción, por parte de los trabajadores, de su espíritu de pertenencia, compromiso y productividad (ISO2600, 2010). Así pues, los psicólogos tienen aquí un campo importante de acción; empero, la evaluación de la responsabilidad social no ha sido una ruta seguida por muchos colegas. Se requiere aquí, de un trabajo interdisciplinario.

\section{La rotación de personal}

Ahora bien, de conformidad con Price Waterhouse Consulting en su informe Saratoga (2013), México es el país de América Latina con el mayor porcentaje anual de rotación de Personal (17.8\%) mientras en América Latina fue de 11.2\%; en Europa, 10\%, y en Estados Unidos, 8.4\%. Esta es un área de oportunidad muy importante para los psicólogos si diseñan, proponen, ponen en marcha y evalúan los resultados de programas para elevar el compromiso de los trabajadores a fin de permanecer en su organización, con la consiguiente disminución en los costos, pues el porcentaje de renuncias voluntarias fue de $9.2 \%$ y el de

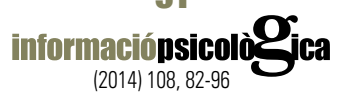


salidas involuntarias 5.8\% (No hay explicación en el informe respecto a la razón por la cual estos dos porcentaje no llegan al total informado previamente de 17.8\%). Si las separaciones involuntarias fueron por no cumplir los trabajadores con las expectativas del puesto, entonces se presenta otra posibilidad de intervención para los psicólogos: mejorar los procesos de selección con instrumentos diseñados para evaluar las competencias requeridas en el trabajo. Y, si fueron voluntarias, se implica la necesidad de incrementar el compromiso de los trabajadores.

\section{El estrés}

De acuerdo con un informe de la revista Expansión (2012), por otro lado, cuatro de cada diez ejecutivos nacionales consideró un incremento en su tensión en el trabajo debido a "causas tales como los conflictos internos en la oficina, las políticas de trabajo y tratar de alcanzar las metas de rendimiento". Desde luego, no son comunes los métodos para identificar los costos del estrés organizacional en el Personal, ya que permanecen ocultos. Quizá se refleja este fenómeno en el ausentismo y la rotación externa, situaciones no contabilizadas generalmente en las organizaciones. Empero, esta es otra área de oportunidad para los psicólogos: establecer y evaluar programas preventivos y correctivos al respecto para elevar la calidad de vida.

\section{Los Estados de Capital Humano}

Otra área de oportunidad para los psicólogos y colegas afines estriba en cooperar con los contadores para enriquecer los estados financieros con estados de Capital Humano (índices de rotación, de capacitación, satisfacción con el trabajo, los jefes, los compañeros y las políticas de la organización; compromiso, inversiones [no gastos] en capacitación, retorno sobre las inversiones en capital humano, edades, escolaridades, salud física y mental, etc., de los trabajadores) De esta manera, apoyarán en la planeación estratégica de las organizaciones y auxiliarán a los directivos a enfocar mejor las decisiones.

Realmente, es necesario considerar que, desgraciadamente, las tasas de empleo informal son enormes en México. Se estima que un $65 \%$ de la población económicamente activa se encuentra en la informalidad. El Gobierno, en vez de promover la creación de empleos, la obstaculiza. Por ejemplo, en las últimas reformas fiscales de este año 2014 ha eliminado la deducibilidad de varias prestaciones otorgadas por las empresas a sus trabajadores, elevando así el costo de los empleos formales y fomentando la informalidad. No obstante, de acuerdo a las estimaciones de la Organización para la Cooperación y el Desarrollo Económico (2013), el 8.2\% de las personas empleadas en México estaban en el sector agrícola, el $26.7 \%$ en la industria y el $64.1 \%$ en los servicios. Nuevamente, se presenta la oportunidad de diseñar instrumentos para valorar la disposición de los candidatos para el trato con las personas y su actitud de servicio.

\section{El compromiso}

Ahora debo volver al tema del compromiso. De acuerdo con una investigación llevada a cabo por la firma de consultoría Towers Watson (2014), el 63\% de las empresas mexicanas

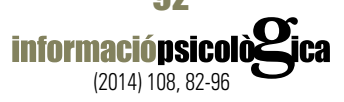


tiene problemas para retener a los empleados con habilidades importantes para la propia organización; una cifra superior al promedio mundial (56\%). Igualmente, hubo manifestaciones de dificultad para obtener la permanencia de quienes presentan un alto potencial y un desempeño sobresaliente (muy probablemente porque son el blanco de los "cazadores de talentos"). En esa misma investigación se encontró un dato importantísimo: entre 50 empresas mundiales "aquellas con una fuerza laboral con un compromiso sustentable alto tenían márgenes anuales tres veces superiores a aquellas con una fuerza laboral no comprometida" (p. 1).

Debe considerarse aquí la posibilidad de reflejarse en estos datos la nueva composición de la fuerza de trabajo. Existe una tendencia mundial hacia el hedonismo y la llamada "post modernización" por Inglehart (1997) en la cual el énfasis se aleja de los bienes materiales y de supervivencia y se acerca a otros valores, tales como la autonomía, la libertad, la participación en la toma de decisiones y el logro del bienestar subjetivo, lo cual es más notorio en los países industrializados y las personas jóvenes. México se encuentra en el medio, según Delhey (2009). Así pues, los psicólogos deberán ayudar a las organizaciones a establecer sistemas de retención del personal altamente capacitado y joven.

Ahora bien, según la Organización Internacional del Trabajo (International Labour Organization, 2014), las previsiones de la tasas de desempleo en México estarán alrededor del 5\% entre 2015 y 2016 (las cuales no son diametralmente diferentes de las experimentadas en los últimos años), lo cual implica una posible oportunidad para los psicólogos, puesto que sería un indicio de la necesidad de selección de personal: pese a las altas tasas de rotación, el empleo se mantendrá. Un desafío fundamental para los psicólogos y las empresas será elevar el compromiso de los trabajadores.

Muy importante resulta traer a colación una nota adicional para indicar la perspectiva de los ejecutivos mexicanos de mayores contrataciones de Personal en 2015; así lo comentó el 53\% de los altos directivos entrevistados por la consultoría Price Waterhouse (2014). Al mismo tiempo, el 56\% demandó al Gobierno potenciar la mano de obra calificada y el 45\% le indicó la necesidad de crear empleo para los jóvenes de 16 a 24 años. Estas voces optimistas indican coyunturas para los psicólogos.

\section{Las innovaciones tecnológicas}

Otro punto necesario para tomar en cuenta se refiere a los adelantos tecnológicos, los cuales tendrán una influencia marcada en la forma de conducir las organizaciones. Por ejemplo, serán cada vez más comunes los equipos virtuales de trabajo, donde los integrantes podrán estar localizados en diversas partes del mundo. Igualmente, ya en México y otros países de Latinoamérica, el trabajo en casa será cada vez más frecuente. En este caso, se requiere todavía más el compromiso debido a una situación característica de este tipo de labores: cada persona labora a su propio ritmo, sin supervisión directa. De la misma manera, es preciso aprovechar las nuevas tecnologías en el área de capacitación.

No debe pasarse por alto el cada vez más difundido empleo de robots en las fábricas, los bancos y otras organizaciones, con el consiguiente desplazamiento de personas. Se necesita, 
entonces, fomentar la adquisición de competencias para diseñar, fabricar y dar mantenimiento a dichos mecanismos. Insisto nuevamente, los psicólogos no deben dejar de pasar esta oportunidad para trabajar con otros profesionales a fin de acelerar el aprendizaje y el reciclaje de las personas.

Inclusive se afirma constantemente la entrada a la sociedad del conocimiento; empero, la práctica no ha ampliado dicho conocimiento en el campo de la Psicología del Trabajo y las Organizaciones en México en áreas importantes, tales como la medición de competencias ligadas a las organizaciones de todo tipo, las repercusiones de la capacitación, los factores del comportamiento ligados a los accidentes de trabajo, el compromiso hacia la organización, la calidad de vida en el trabajo y así sucesivamente, y mostrar mediante los índices de beneficio/costo la valía de la intervención profesional de los psicólogos. Por ende, se requiere urgentemente de una nueva visión en los psicólogos y colegas afines: dejar de lado las adoradas y tradicionales perspectivas y fijar la mirada en los cambios avizorados para el futuro tomando parte activa en ellos.

\section{Factores favorables}

Sí, estas impresiones manifiestan un marcado tinte pesimista. Sin embargo, parecen aproximarse vientos favorables. En efecto, de acuerdo con una nota de la revista Expansión (2014a), en una investigación llevada a cabo por el Conference Board en Latinoamérica, el capital humano constituye una de las principales preocupaciones de los directivos, al unísono con la excelencia operativa, el desarrollo de la innovación, el acercamiento a los clientes y las regulaciones gubernamentales. Así pues, si estas preocupaciones conducen a prestar mayor atención y recursos a las primeras cuatro mencionadas, los psicólogos pueden llevar a cabo grandes aportaciones, si se desprenden de las acciones y los puntos de vista tradicionales (como los ilustrados por las anécdotas mencionadas previamente) y se enfocan en determinar la manera en la que pueden (y deben) contribuir con efectividad al incremento en la productividad y la calidad de vida. Traigo a colación, por enésima vez, la necesidad de mostrar las repercusiones financieras de las acciones relativas al Capital Humano.

Otro punto interesante: según una encuesta sobre "Las mejores universidades de México" (2014), el 41\% de los egresados en el campo de la Psicología tenía entre 25 y 34 años, con un promedio mensual aproximado de $\$ 8,540.00$ (alrededor de 660 dólares norteamericanos); el $78 \%$ eran mujeres; y el $58 \%$ trabajaba en la industria privada. Parece, entonces, existir una buena acogida de los egresados de esta carrera en el campo de la iniciativa privada, lo cual conlleva la responsabilidad de brindar frutos positivos de sus labores.

\section{Recomendaciones:}

Así pues, desde mi ángulo personal, para el futuro sugiero:

1. Diseñar y evaluar herramientas de detección de competencias específicas para cada tipo de trabajo (por caso, labores relacionadas con el tratamiento de información por medios computacionales, atención a los clientes, labores ingenieriles, etc.) en vez de emplear tests generales.

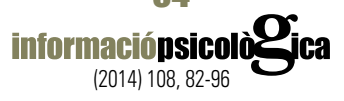


2. Mejorar la detección de necesidades de capacitación, igualmente para cada tipo de trabajo, en vez de ofrecer sólo cursos generales (Relaciones Humanas, Calidad y otros demasiado amplios). Nuevamente, no confundir dar cursos o llevar a cabo dinámicas de grupo con capacitar. (En mi libro de 1994 presento un sistema para lograr este desiderátum).

3. Acercarse más a los aspectos administrativos, especialmente en lo relativo a los índices de beneficio/costo de todas las acciones profesionales, con la finalidad de mostrar la valía de nuestro trabajo. Insisto en un punto: si exhibimos nuestras aportaciones desde el ángulo financiero, estaremos comprando un seguro de desempleo.

4. Dirigir la atención a áreas poco atendidas por los psicólogos hasta ahora, tales como la cultura de calidad, los equipos autodirigidos, la responsabilidad social, la higiene y la seguridad, el trabajo en casa y otras semejantes.

5. Como continuación del punto 3, adoptar una postura de evaluación y no sólo de acción. En tanto no podamos responder a preguntas fundamentales, tales como: ¿cuáles son las aportaciones de tu trabajo como psicóloga(o) a la efectividad de la organización? ¿estamos invirtiendo o desperdiciando en las funciones relativas al Personal? ¿cuál es el beneficio/costo de tus acciones cotidianas? y otras semejantes no se considerarán indispensables las tareas relativas al Capital Humano.

\section{Referencias}

Albín, O. (2013). Mano de obra más capacitada para automotriz. Recuperado de file:///C:/Users/myrcha/GRUPO HOGAR/RH/Mano obra capacitada automotriz.htm

Arias Galicia, F. (1994). Capacitación para la competitividad y la colaboración. México: Asociación Mexicana de Capacitación de Personal.

Arias Galicia, F. (2010). Metodología de la investigación. 7a. ed. México: Trillas.

Banco Interamericano de Desarrollo (2014). Desconectados. Recuperado de http://www.iadb.org/es/videos/ ver,2173.html?videolD=9629\&

Cascio, W. (1999). Costing Human Resources. The financial impact of human behavior in organizations. Boston: Cengage learning.

Centro de Investigación para el Desarrollo, A.C. (2014). Encuesta de competencias profesionales. México: autor.

Delhey, J. (2009). From materialist to postmaterialist happiness? National affluence and determinants of life satisfaction in cross-national perspective. World Values Re-search 2(2), 30-54.

Expansión (2012). Empleados mexicanos entre los más tensos. Recuperado de http://www.cnnexpansion.com/ mi-carrera/2012/02/08/empleados-mexicanos-entre-los-mas-tensos.

Expansión (2014a). Competitividad, preocupación de los CEO. Recuperado de

http://www.cnnexpansion.com/mi-carrera/2014/01/27/que-le-preocupa-al-ceo-en-al

Expansión (2014b). Ejecutivos ven más contrataciones. Recuperado de http://www.cnnexpansion.com/expansion/2014/04/30/ejecutivos-ven-mas-contrataciones 


\section{praxis}

Estado actual de la Psicología del Trabajo y las Organizaciones en México: un ensayo crítico.

Expansión (2014c). Súper empresas Expansión. 2014. Recuperado de http://www.cnnexpansion.com/tablas/2014/05/20/super-empresas-2014-mas-de-3000-empleados?newsvcnn2=1402488273

Gran Thorton (2014). International business report. Recuperado de https://dataviztool.internationalbusinessreport.com/ibr.html\#map/a-lack-of-availability-of-a-skilled-workforce/quarterly.

Inglehart, R. (1997). Modernization and postmodernization. Cultural, economic, and political change in 43 societies. Princeton, N.J.: Princeton University. Press.

International Labour Organization (2014). Global employment trends. Autor: Ginebra.

Juárez García, A. (2014) Investigaciones psicométricas de factores psicosociales en trabajadores mexicanos. México: Plaza y Valdés.

ISO 26000:2010. Recuperado de https://www.iso.org/obp/ui/\#iso:std:iso:26000:ed-1:v1:en.

Las mejores universidades de México. (2014). Recuperado de http://mejoresuniversidadesdemexico.mx/sites/ default/files/pdfs/mejores 2014/\#/78/zoomed

López-Acevedo, G., Tinajero, M. y Rubio, M. (2005). Mexico: human capital effects on wages and productivity. World Bank Policy Research Working Paper 3791. Washington: Banco Mundial.

Organización para la Cooperación y el Desarrollo Económico. (2013). OECD Labour Statistics. 2003-2012. Recuperado de http://www.keepeek.com/Digital-Asset-Management/oecd/employment/oecd-labour-force-statistics-2013_oecd_Ifs-2013-en

Price Waterhouse Consulting. (2013). Saratoga 2012. Efectividad del capital humano en América Latina. Reporte Ejecutivo. México: Autor. 2

Price Waterhouse Consulting. (2014). Confianza y transformación: México explora nuevas realidades económicas., México: Autor.

Quintini, G. (2014). Skills at Work: How skills and their use matter in the labour market, OECD Social, Employment and Migration Working Papers, No. 158. Paris: OECD Publishing. http://dx.doi. org/10.1787/5jz44fdfjm7j-e

Rojas Sierra, D. N. y Acevedo Rocha, E. A. (1998). ¿Universal la norma del Cleaver?. Resultados de una investigación hecha con profesores mexicanos. Instituto Tecnológico y de Estudios Superiores de Monterrey, Campus Ciudad de México.

Towers Watson. (2012). Estudio global de la fuerza laboral. Recuperado de http://www.towerswatson.com/esMX/Insights/IC-Types/Survey-Research-Results/2012/07/2012-Towers-Watson-Global-Workforce-Study 